\title{
A MUDANÇA NO CICLO FAMILIAR DIANTE DA SÍNDROME DO NINHO VAZIO: UMA REVISÃO
}

Fernando Silvio de Souza Virgolino. Discente do Curso Bacharelado em Enfermagem da Universidade Federal de Campina Grande - Campus - Cuité/Paraíba/Brasil. E-mail: fernandovirgolino@ hotmail.com.

Amanda Bezerra da Silva. Discente do Curso Bacharelado em Enfermagem da Universidade Federal de Campina Grande - Campus - Cuité/Paraíba/Brasil. E-mail: amanda_bezerra05@ hotmail.com

Sarah Coêlho de Araújo Silva Porto. Discente do Curso Bacharelado em Enfermagem da Universidade Federal de Campina Grande - Campus - Cuité/Paraíba/Brasil.: E-mail: sarahcoelhotche@gmail.com

Vinicius Lino de Souza Neto. Discente do Curso Bacharelado em Enfermagem da Universidade Federal de Campina Grande - Campus - Cuité/Paraíba/Brasil. E-mail: vinolino@ hotmail.com

Alynne Mendonça Saraiva. Enfermeira. Doutoranda em Enfermagem pela Universidade Federal da Paraíba - UFPB. Mestre em Enfermagem pela Universidade Federal da Paraíba/UFPB/PB/BRASIL. Docente do Curso de Bacharelado em Enfermagem da Universidade Federal de Campina Grande - UFCG/ Campus - Cuité. E-mail:

alynnems@hotmail.com

\section{Recebido em: 15/05/2013 - Aprovado em: 30/07/2013 - Disponibilizado em: 15/08/2013}

\section{RESUMO}

Objetivos: explanar as mudanças ocorridas no âmbito familiar em decorrência Síndrome do Ninho Vazio. Método: estudo exploratório e descritivo, realizado por meio de revisão integrativa da literatura, utilizando publicações científicas disponibilizadas nas bases de dados LILACS, Medline e SciELO, entre os anos de 2004 a 2012 , tendo como amostra final 08 artigos. Resultados: a pesquisa aponta a deficiência de publicação frente a temática em questão, pois é substancial entender e/ou conhecer a Síndrome do Ninho Vazio, sua etiologia, comorbidades e o impacto que a mesma causa no ambiente familiar. A maioria dos artigos foi produzida por profissionais psicólogos, evidenciando um desconhecimento ou desinteresse por parte de outros profissionais em abordar tal assunto. Conclusão: Diante disto, a Síndrome do Ninho Vazio se expressa como um transtorno de tempos contemporâneos, afetando diretamente as relações familiares, sendo necessário maiores pesquisas na temática para a divulgação da síndrome e a abordagens de novas estratégias de enfrentamento.

Descritores: Síndrome do Ninho Vazio. Pais. Meia- Idade. Relações familiares. Estágios do Ciclo Vital.

\section{Change in family before the cycle of empty nest syndrome: a review}

\begin{abstract}
Objectives: To explain the changes in the family due Empty Nest Syndrome. Method: exploratory and descriptive study, conducted through integrative literature, using scientific publications available in the databases LILACS, SciELO and MEDLINE between the years 2004 to 2012, with the final sample of 08 articles. Results: The research points to the deficiency of the front thematic publication in question, it is substantial to understand and / or know the Empty Nest Syndrome, etiology, co-morbidities and the impact that it causes in the family environment. Most articles were produced by professional psychologists, revealing ignorance or disinterest on the part of other professionals in addressing this issue. Conclusion: Given this, the Empty Nest Syndrome is expressed as a disorder of contemporary
\end{abstract}


times, directly affecting family relationships, necessitating further research on the topic for the dissemination of new approaches syndrome and coping strategies.

Keywords: Empty Nest Syndrome. Parents. Middle Aged. Family relationships. Stages in the Life Cycle.

\section{INTRODUÇÃO}

O ciclo vital faz parte da composição orgânica e funcional do ser humano, círculo este, que se divide nas seguintes fases: período de crescimento, maturidade e por fim o de declínio. Estas fases são caracterizadas por perdas biológicas e sociais e marcadas pelas transições na pirâmide familiar (FARIA \& SEIDL, 2005). Na etapa de declínio podem ser observados sinais de depressão, dependência e desestruturação familiar, sintomas associados a Síndrome do Ninho Vazio, ou seja, esses sentimentos brotam quando ocorre a perda do papel da função parental, com a saída dos filhos da casa de seus pais (SARTORI \& ZILBERMAN, 2009).

A sociedade vive em crise de valores e ideologias constantemente, proporcionando uma instabilidade presente no nosso cotidiano, o reflexo dessas mudanças é observável em diversas instituições, a família é uma delas (BAUGMART \& SANTOS, 2009). Dentro desta perspectiva, vem se constatando que o ciclo evolutivo da família brasileira está sofrendo alterações, aonde cada vez mais cedo os filhos vão à busca da sua independência financeira, ou, em busca de uma relação conjugal precocemente, deixando assim um vazio dentro do lar de convívio com os pais. De acordo com Oliveira (2007), quando o último filho sai de casa ou ocorre a morte de um dos parceiros conjugais, este período pode ser susceptível para o surgimento da Síndrome do ninho vazio.

Alguns autores enfatizam que o sexo feminino está mais vulnerável a apresentar sintomas desta síndrome, pois a mulher perde o seu papel fundamental na base familiar, o de cuidadora (TAVARES et al., 2004). Mulheres que se dedicaram a maior parte da sua vida à criação dos seus filhos de modo exclusivo sofrem ao vê-lo partindo, gerando para si um autoconceito "impotência", refletindo assim em uma auto-estima baixa, favorecendo um quadro depressivo e até mesmo a reclusão do convívio social (WEBBER \& DELVIN, 2010).

Entretanto, pesquisas recentes mostram que a figura paterna está também vulnerável ao acometimento pela Síndrome do ninho vazio, prevalecendo em sua maioria um sentimento de acomodação, refletindo assim conflitos entre os casais. Neste mesmo segmento, o estudo mostra que as mulheres podem se encontram mais disponíveis para se lançarem nos seus projetos, aos quais protelou para se dedicar a criação dos filhos, enquanto 
entre os homens o sentimento pode ser contrário, pois passam a se dedicar as tarefas domésticas (HOBDY, et al., 2007).

Diante do exposto, este estudo tem como objetivo fazer uma revisão integrativa que retrate as mudanças que ocorrem no seio familiar decorrente da síndrome do ninho vazio.

\section{METODOLOGIA}

Para atingir o objetivo proposto foi realizada uma pesquisa documental indireta, sendo descritiva exploratória, do tipo revisão integrativa da literatura, na busca de estudos literários nacionais e internacionais sobre publicações relativas ao tema, operacionalizada pelas etapas de formulação do problema, coleta de dados, avaliação dos dados, análise e interpretação dos dados coletados e apresentação dos resultados. A revisão integrativa segundo Souza, Silva, e Carvalho (2010), determina o conhecimento atual sobre uma temática específica, já que é conduzida de modo a identificar, analisar e sintetizar resultados de estudos independentes sobre o mesmo assunto, contribuindo, pois, para uma possível repercussão benéfica ao tema estudado.

Ademais, possibilita a divulgação do conhecimento, pois um único estudo disponibiliza ao leitor os resultados de várias pesquisas (MENDES; SILVEIRA; GALVÃO, 2008). Convém ressaltar que o estudo teve o intuito de responder a seguinte questão norteadora: Quais as mudanças que ocorre no seio familiar decorrente da Síndrome do Ninho Vazio?

A identificação das publicações foi mediada, inicialmente, por meio da busca nas seguintes bases de dados indexadas na Biblioteca Virtual em Saúde (BVS): Literatura Latino-Americana e do Caribe em Ciências da Saúde (LILACS), Medical Literature Analysisand Retrieval Sistem online (Medline), Scientific Electronic Library Online (SciELO).

No primeiro momento, utilizaram-se como descritores, tanto em português como em inglês: Síndrome do Ninho Vazio/Empty Nest Syndrome; Pais/ Parents; Meia-Idade/ Middle Aged; Relações Familiares/ Family Relations; Estágios do Ciclo Vital/ Stages in the Life Cycle. Após a seleção, os artigos foram lidos na íntegra e elencados de acordo com os seguintes critérios de inclusão: periódicos publicados em português e inglês com títulos, resumos e textos completos, coerentes com a temática proposta, publicados no período entre 2004 a 2012. Durante a seleção, alguns artigos foram excluídos conforme alguns critérios, na quais são: trabalhos que abordassem outros tipos de temática, produções cientifica com animais experimentais, trabalhos direcionados a crianças e pôr fim artigos que não tinham o português e inglês como idioma. 
A busca foi efetivada nos meses de fevereiro e março 2013, e as estratégias utilizadas para o levantamento dos artigos foram adaptadas para cada uma das bases de dados, de acordo com suas especificidades de acesso, tendo como eixo norteador a pergunta e os critérios de exclusão. A análise criteriosa das publicações possibilitou a obtenção das informações mais relevantes, assim 08 artigos constituíram a amostra final.

A localização dos artigos, corpus do estudo, foi realizada de forma independente, como estratégia de garantir a legitimidade do conteúdo de análise (POLIT; BECK; HUNGLER, 2006). A fim de analisar os dados utilizou-se a análise textual, a qual possibilita uma discussão sobre a temática pesquisada embasada na literatura atualizada pertinente.

\section{RESULTADOS E DISCUSSÃO}

Os dados a seguir referem-se ao levantamento de artigos do estudo em questão de acordo com os critérios de seleção elaborados pelos pesquisadores para realização da analise do material referente à temática. Foi possível observar durante buscas aos periódicos supracitados, que ainda existe uma deficiência de produção cientifica a nível nacional e internacional frente à contextualização da síndrome do ninho vazio e o seu reflexo no âmbito familiar. Com isso, no processo de operacionalização do presente estudo foram elencadas diversas variáveis referentes às características dos autores da população amostral, através de tabelas sinópticas, conforme explana a Tabela - 1, logo abaixo:

Tabela 1 - Distribuição das características dos tipos de estudos que fizeram parte desta revisão, elencados entre os anos de 2004 a 2012, quanto ao tipo de estudo.

\begin{tabular}{lcr}
\hline TIPO DE ESTUDO & N & \% \\
\hline Estudo de campo & 02 & 25,00 \\
Revisão da Literatura & 03 & 37,50 \\
Estudo Transversal & 01 & 12,50 \\
Retrospectivo descritivo & 02 & 25,00 \\
Total: & 08 & 100,00
\end{tabular}

Fonte: Dados da Pesquisa, 2013.

Conforme foi analisado, a Tabela-1, os periódicos usados para o desenvolvimento do referente estudo obtiveram-se com maior percentual, pesquisas do tipo revisão da literatura $(37,5 \%)$, em seguida aparecem os estudos de campo (25\%), retrospectivo descritivo (25\%) e um único estudo de teor transversal $(12,50 \%) \quad$ totalizando o quantitativo da amostra.

De um modo geral, conforme os resultados supracitados, mesmo tendo um pequeno número de produções relacionado a temática, em sua maioria se configuram com revisão de literatura embasadas em livros e periódicos publicados nas décadas de 80 á 90. Mostra-se então a necessidade de pesquisas voltadas para as mudanças sociais e psíquicas 
provocadas pela síndrome, bem como estudos que possam lançar novas estratégias de cuidado para esse publico peculiar.

$\mathrm{Na}$ segunda tabela, podemos observar que esse é um tema relativamente recente no meio científico. A revisão aponta que os estudos se iniciaram com maior intensidade há quase uma década. Pode-se atrelar a este fato, a repercussão que os estudos relativos a temática da saúde mental nos últimos dez anos ter ganhado uma maior notoriedade em todo mundo.

Tabela 2 - Distribuição das características dos periódicos que fizeram parte desta revisão, elencados entre os anos de 2004 a 2012, quanto ao ano e tipo de revista.

\begin{tabular}{|c|c|c|c|c|c|}
\hline \multicolumn{6}{|c|}{ Variáveis } \\
\hline Ano & $\mathrm{N}$ & $\%$ & Revista & $\mathrm{N}$ & $\%$ \\
\hline 2009 & 01 & 12,5 & Centro & 01 & 12,5 \\
\hline & & & $\begin{array}{l}\text { Terapia de Casal } \\
\text { e Família }\end{array}$ & & \\
\hline 2005 & 01 & 12,5 & $\begin{array}{l}\text { Psicologia: } \\
\text { reflexão e crítica }\end{array}$ & 01 & 12,5 \\
\hline 2004 & 04 & 50 & $\begin{array}{l}\text { Revista Brasileira } \\
\text { de Psiquiatria }\end{array}$ & 04 & 50 \\
\hline 2007 & 01 & 12,5 & Int $\mathrm{J}$ Aging Hum & 01 & 12,5 \\
\hline 2007 & 01 & 12,5 & Colloquium & 01 & 12,5 \\
\hline & & & Humanarum & & \\
\hline Total: & 08 & 100 & Total: & 08 & 100 \\
\hline
\end{tabular}

Fonte: Dados da Pesquisa, 2013.

No processo de avaliação do quantitativo amostral, foram elencadas as variáveis quanto ao ano e a revista em que o artigo foi publicado. Percebe-se uma predominância de publicações do ano de
2004(50\%) e da Revista Brasileira de Psiquiatria (50\%). Foram encontrados também artigos em diversos anos de publicação e revistas, tanto de cunho nacional como internacional. Embora a maioria das publicações tenha sido feita através da Revista Brasileira de Psiquiatria, foi observado que as pesquisas se deram em sua maioria na área de psicologia.

Isso se reflete devido ao maior número de autores dos artigos terem formação em psicologia, como mostra na tabela - 3 a seguir:

Tabela 3 - Distribuição das características dos periódicos que fizeram parte desta revisão, elencados entre os anos de 2004 a 2012, quanto a formação dos autores que publicaram nos periódicos.

\begin{tabular}{lcr}
\hline Autores da Publicação & N & \% \\
\hline Psicólogo & 05 & 62,5 \\
Enfermeiro & 01 & 12,5 \\
Psiquiatra & 02 & 25 \\
Total: & 08 & 100 \\
\hline
\end{tabular}

Fonte: Dados da Pesquisa, 2013.

Observou-se na Tabela -3 , os maiores pesquisadores que aborda a Síndrome do Ninho Vazio, são psicólogos (62,5\%), consecutivamente temos Enfermeiro (a) $(12,5 \%)$ e por fim os psiquiatras $(25 \%)$, isso sumariza que a área é pouco explorada pelos psiquiatras e enfermeiros. ̀̀ importante 
destacar que se trata de uma síndrome que vem aumentando suas vítimas nos últimos anos, e muitas vezes por seus sintomas serem semelhantes a outros transtornos mentais, torna-se difícil seu diagnóstico, necessitando de um maior aprofundamento científico.

Além disso, pode-se estabelecer outra discussão a respeito desse resultado, já que por se tratar de uma síndrome que se configura inicialmente nos aspectos sociais da vida para depois se estabelecer como patologia psíquica, é sabido que a medicina ainda focaliza de maneira mais intensa os sintomas da doença, favorecendo a psiquiatrização e medicalização dos problemas sociais.

\section{CONSIDERAÇÕES FINAIS}

Conforme análise realizada de todos os periódicos nacionais e internacionais, percebe-se uma deficiência de publicações que abordem a temática em questão, assunto este, de grande importância para ser investigado, pois se vive em um tempo onde o sofrimento mental torna-se mais presente e evidente em uma população onde os valores humanos estão sendo transformados a cada dia.

No transcorrer do estudo fica evidente que pesquisas voltadas para a síndrome no ninho vazio ainda são incipientes, e que somente há uma década percebe-se o aumento de interesse pela temática. Esse fato pode estar associado a fato das pesquisas no âmbito da saúde mental estar ganhando uma maior notoriedade no mundo acadêmico, principalmente os estudos que se voltam não somente ao modelo hospitalocêntrico e focalizado nos sinais e sintomas da doença.

Ao perceber que as publicações estão voltadas também para as transformações familiares decorrentes desta síndrome, observa-se que os pesquisadores estão explorando as implicações sociais trazidas pela presença do sofrimento mental em membros da família.

Fica evidente que a Síndrome do Ninho Vazio é um problema não somente de cunho psíquico, mas também social, aonde a sua prevalência e incidência vem se tornando evidente no campo dos saberes científicos, pois antes, as correntes literárias atrelava-se a patologia ao sexo feminino, hoje, já se sabe que não existe mais essa discrasia, pois a estrutura familiar vem mudando consideravelmente em todos só seus aspectos.

Diante disso, fica a emergência de novas estratégias de cuidados que possam dar suporte a família e projetos sociais que possam incluir as pessoas da terceira idade, evitando a perca da funcionalidade $\mathrm{e}$ isolamento social, fatores propensos para o desencadeamento de sofrimento. 


\section{REFERÊNCIAS}

BAUMGART, J. A. R.; SANTOS, D. L. Síndrome do Ninho vazio: possíveis considerações. DOMUS - Centro de Terapia de Casal e Família. Porto Alegre. v. 13, n.1, p. 93 - 101, 2009.

Disponível em:

https://psicologia.faccat.br/moodle/pluginfile.php/197/course/section/101/camilas.pdf Acesso em: 05 mar.2013.

FARIA, J. B.; SEIDL, E. M. F. Religiosidade e enfrentamento em contexto de saúde e doença: revisão da literatura. Psicologia: reflexão e crítica. 18(3), p. 381-389, 2005. Disponível em: http://www.scielo.br/pdf/prc/v18n3/a12v18n3.pdf Acesso em: 05 mar.2013.

HOBDY et al. The role of attachment style in coping with job loss and the empty nest in adulthood. Int J Aging Hum Dev. 65(4): 335-71, 2007. Disponível em: http://www.scielo.br/scielo.php?script=sci_arttext\&pid=S0101-60832009000300005 Acesso em: 05 mar.2013

MENDES, K.D.S.; SILVEIRA, R.C.C.P.; GALVÃO, C.M. Revisão integrativa: métodode pesquisa para a incorporação de evidências na saúde e na enfermagem. Texto Contexto Enferm.17(4): 758-64, 2008.Disponível em: .

http://200.144.190.38/bitstream/handle/2012.1/3509/art_MENDES_Revisao_integrativa_metod o_de_pesquisa_para_a_2008.pdf?sequence=1. Acesso em: 05 mar.2013

OLIVEIRA, A. Adolescência prolongada: Um olhar sobre a nova geração. Colloquium Humanarum. São Paulo, v. 4, n. 1, p. 31-45, jun. 2007. Disponível em: http://revistas.unoeste.br/revistas/ojs/index.php/ch/article/viewFile/224/602Acesso em: 05 mar.2013.

POLIT,D.F.; BECK, C.T; HUNGLER, B.P. Essentials of nursing research: methods, appraisal and utilization. Philadelphia: Lippincott Williams \& Wilkins; 2006. Using research in evidencebased nursing practice; p. 457-94.

SOUZA, M.T.; SILVA, M.D; CARVALHO, R. Revisão integrativa: o que é e como fazer. Einstein. 8(1 Pt 1):102- 6, 2010. 
SARTORI, A. C. R.; ZILBERMAN, M.L. Revisando o conceito de ninho vazio. Rev Psiq Clín. 36(3): 112-21, 2009. Disponível em:

http://www.scielo.br/scielo.php?script=sci_arttext\&pid=S01016083200900-0300005. Acesso em: 05 mar.2013.

TAVARES, M.B et al. Características de comportamento do filho único vs filho primogênito e não primogênito. Revista Brasileira de Psiquiatria, n. 1, v. 26, p. 17 - 23, 2004. Disponível em: http://www.scielo.br/scielo.php?script=sci_arttext\&pid=S1516-44462004000100007 Acesso em: 05 mar.2013.

WEBBER, C.; DELVIN, D. Empty-nest syndrome. Set. 2010. Disponível em: http://www.netdoctor.co.uk/womenshealth/featores/ens.html. Acesso em: 05 mar.2013. 